\title{
THE POTENTIAL OF THE NSW POISONS INFORMATION CENTRE FOR SURVEILLANCE OF UNINTENTIONAL POISONING IN YOUNG CHILDREN
}

\section{David Muscatello \\ Epidemiology \& Surveillance Branch \\ NSW Department of Health \\ Greg Saville \\ National Poisons Register}

This article reports information collected routinely by the NSW Poisons Information Centre (NSW PIC), and comments on its potential to enable the monitoring of episodes of unintentional poisoning in NSW children. Unintentional poisoning is a major cause of morbidity in infants and young children. In NSW in 1995-96, unintentional poisoning was the second most common cause of injury-related hospitalisation in children aged under five years. ${ }^{1}$ Despite this, there is a dearth of information in NSW describing the substances and risk factors involved in serious poisoning.

The NSW PIC provides expert advice on the appropriate management of poisoning, and the handling of toxic substances, to both the general public and clinicians. The service is available to the population of NSW 24 hours a day, seven days a week. Information collected by the NSW PIC may be useful in informing poisoning prevention activities and for setting prevention priorities.

\section{METHODS}

The NSW PIC maintains a database of information describing each phone enquiry (or 'exposure call') for assistance with the management of a person who has ingested or been exposed to a toxic substance. Information on 'exposure calls' originating from NSW for patients aged 0 to 4 years was extracted from the database for the 1996 calendar year.

Information collected included:

- the substance involved

- patient age and sex

- route of exposure (ingestion, inhalation, skin, eyes)

- time since exposure

- initial symptom assessment

- the outcome of the call (or 'call handling').

Circumstantial information, such as the packaging type or means of access to the substance is not routinely collected. The severity of the exposure is not explicitly recorded in the database. However, the outcome of the call (or the 'call handling') provides a proxy indication of the severity of the poisoning. The advice given is categorised as:

- stay home

- refer to general practitioner

- refer to hospital
- remain in hospital.

Where the advice is 'refer to hospital', the exposure is potentially more serious than those advised to remain at home or consult a general practitioner. Where a call originates from a hospital or general practitioner, no additional information about the call outcome is recorded.

This analysis was restricted to the toxic substance involved and the advice given, as these were considered to be the most relevant for informing prevention activities in NSW. Data were analysed using SAS statistical software. ${ }^{2}$

\section{RESULTS}

In 1996, the NSW PIC handled 24,707 'exposure calls' that originated in NSW and related to potential exposures to poisoning substances in children aged under five years. Of these, the majority $(20,461$, or 83 per cent) were advised to stay at home, and a small proportion $(1,041$, or four per cent) were referred to hospital. Approximately 10 per cent of calls originated from hospital staff or general practitioners (Table 1).

As shown in Table 2, the most common substances involved in all 'exposure calls' were:

- paracetamol (1,024 calls, four per cent);

- cough-cold preparations containing no paracetamol or aspirin (782, three per cent);

- all-purpose hard surface cleaners (767, three per cent).

Among calls resulting in advice to attend hospital, paracetamol (143, or 14 per cent) and cough-cold preparations containing no paracetamol or aspirin (63, six per cent) remained the most common exposures. Antihistamine exposure, which was the sixth most common among all 'exposure calls', is the third most

\section{TABLE 1}

ADVICE PROVIDEDTO PHONE ENQUIRIES TO THE NSW PIC FOR POISONING EXPOSURES IN CHILDREN AGED UNDER FIVE YEARS, 1996.

\begin{tabular}{|lrr|}
\hline 'Call handling' & No. calls & $\%$ \\
\hline Stay home & 20,461 & 82.8 \\
Refer to general practitioner & 278 & 1.1 \\
Refer to hospital & 1,041 & 4.2 \\
Already at hospital & 1,965 & 8.0 \\
Already at general practitioner & 434 & 1.8 \\
Other & 84 & 0.3 \\
Not stated & 444 & 1.8 \\
\hline Total & 24,707 & 100.0 \\
\hline
\end{tabular}




\section{TABLE 2}

TEN MOST COMMON EXPOSURES, ALL CALLS TO NSW PIC COMPARED WITH CALLS REFERRED TO HOSPITALS, CHILDREN AGED UNDER FIVE YEARS, 1996

\begin{tabular}{|c|c|c|c|c|c|}
\hline \multicolumn{3}{|c|}{ All calls to NSW PIC } & \multicolumn{3}{|c|}{ Calls referred to hospitals } \\
\hline Substance/product & No. calls & $\begin{array}{c}\% \text { of calls } \\
(n=24,707)\end{array}$ & Substance/product & No. calls & $\begin{array}{r}\% \text { of calls } \\
(n=1,041)\end{array}$ \\
\hline Paracetamol & 1,024 & 4.1 & Paracetamol & 143 & 13.7 \\
\hline $\begin{array}{l}\text { Cough/cold preparations not } \\
\text { containing paracetamol/ aspirin }\end{array}$ & 782 & 3.2 & $\begin{array}{l}\text { Cough/cold preparations not } \\
\text { containing paracetamol/ aspirin }\end{array}$ & 63 & 6.1 \\
\hline Cleaner: all purpose/ hard surface & 767 & 3.1 & Antihistamines & 40 & 3.8 \\
\hline Oral contraceptives & 627 & 2.5 & Iron (non-multivitamin) & 30 & 2.9 \\
\hline Silica gel & 580 & 2.3 & Tricyclic antidepressants & 26 & 2.5 \\
\hline Antihistamines & 485 & 2.0 & Eucalyptus oil & 25 & 2.4 \\
\hline Perfume, cologne, aftershave & 484 & 2.0 & Battery: disc/button type & 24 & 2.3 \\
\hline Hand dish detergents & 480 & 1.9 & $\begin{array}{l}\text { Paracetamol/narcotic } \\
\text { combination analgesic }\end{array}$ & 23 & 2.2 \\
\hline Vaporiser fluids and inhalants & 404 & 1.6 & Calcium antagonists & 22 & 2.1 \\
\hline $\begin{array}{l}\text { Toilet bowl cleaner/ deodoriser: } \\
\text { cage type }\end{array}$ & 401 & 1.6 & $\begin{array}{l}\text { Anticholinergic drugs: } \\
\text { other/unknown }\end{array}$ & 17 & 1.6 \\
\hline Total & 6,034 & 24.4 & Total & 413 & 39.7 \\
\hline
\end{tabular}

common substance (40, 3.8 per cent) resulting in advice to attend hospital. The 10 most common substances resulting in all 'exposure calls' accounted for only a quarter of all calls made, reflecting the wide variety of substances available in the community. In comparison, the 10 most common substances resulting in advice to attend hospital accounted for 40 per cent of 'exposure calls' in that category (Table 2), reflecting the smaller subset of substances in the community which have potentially dangerous effects.

\section{DISCUSSION}

Paracetamol is the substance most commonly identified in calls to the NSW PIC relating to the unintentional poisoning of young children. While accounting for less than five per cent of all exposure calls, it accounts for 14 per cent of exposures referred to a hospital. This is consistent with information obtained from the NSW Inpatient Statistics Collection (ISC). In 1995-96, 16 per cent of NSW hospitalisations for unintentional poisoning due to drugs and medicines in children aged under five years were the result of paracetamol poisoning. ${ }^{1}$

Many of the substances commonly leading to NSW PIC inquiries, such as oral contraceptives and cleaning agents, do not generally lead to referral to hospital. When analysing NSW PIC information, the 'call handling' provides a convenient marker of the severity of the exposure, and identifies substances implicated in more serious incidents of poisoning.

The information collected by the NSW PIC does not include information describing the circumstances of the poisoning, such as packaging type and availability, which limits its use for routine surveillance. Further, population-based poisoning rates cannot be calculated because the proportion of all poisonings in NSW covered by the NSW PIC is unknown. The categorisation of 'call handling' presents difficulties because for calls made from hospitals and general practitioners only the call source is recorded, not the call outcome.

Collection of the telephone number of the caller could provide a potential means of obtaining more detailed information through later follow-up. This method has been used in Victoria to obtain detailed risk information for several substances. ${ }^{3-5}$

The other routinely collected source of information that describes non-fatal poisoning in NSW is the NSW ISC. ${ }^{6}$ Information on hospitalisations due to poisoning can be readily obtained, including the age and sex of patients, and population-based rates, ${ }^{1}$ but its usefulness is limited by the difficulty of identifying specific substances involved, and the lack of information about the circumstances of the poisoning event. In particular, the International Classification of Diseases, Clinical Modification, ${ }^{7}$ used in the coding of hospital statistics in NSW, does not adequately permit the distinction of substances involved in poisoning exposures. For example, antihistamine poisoning would be coded under the diagnosis of Poisoning by primarily systemic agents-antiallergic and antiemetic drugs and would be given an external cause of injury code of Accidental poisoning by other drugs-primarily systemic agents. In comparison, the index of substances used by the NSW PIC is based on pharmaceutical categories that are more readily understood in terms of Australian 
prescribing patterns and substances used in Australian homes.

As a result of poisoning there were 933 hospitalisations of children aged under five years in NSW in 1995-96. ${ }^{1}$ For this age group there were more than 3,000 calls to the NSW PIC which were either made from a hospital or which resulted in advice to attend hospital. It is likely, therefore, that the NSW PIC database is adequately representative of most poisoning exposures in this age group.

\section{CONCLUSION}

Of more than 20,000 calls relating to young children in NSW each year, more than 80 per cent result in the advice to 'stay home', thus possibly avoiding a considerable additional burden on health services. The data that is currently collected provides limited but useful information which can assist in setting priorities in poisoning prevention activities, and guide the investigation of risk factors associated with poisoning. The NSW PIC is in a unique position to collect additional specialised poisoning risk information which could enhance prevention activities.

\section{REFERENCES}

1. Public Health Division. The Health of the People of NSWReport of the Chief Health Officer. Sydney: NSW Health Department, 1997.

2. SAS computer program. Version 6.12. Cary (NC): SAS Institute Inc, 1997.

3. Cornish LS, Parsons BJ, Dobbin MD. Automatic dishwasher detergent poisoning: opportunities for prevention. Aust N Z J Public Health 1996; 20: 278-283.

4. Parsons BJ, Day LM, Ozanne-Smith J, Dobbin MD. Rodenticide poisoning among children. Aust N Z J Public Health 1996; 20: 488-492.

5. Day LM, Ozanne-Smith J, Parsons BJ, Dobbin MD, Tibballs J. Eucalyptus oil poisoning among young children: mechanisms of access and the potential for prevention. Aust N Z J Public Health 1997; 21: 297-302.

6. NSW Health Department. The Inpatient Statistics Collection [cited 1999 Sep 29].

URL: www.health.nsw.gov.au/iasd/isc.

7. National Coding Centre. The Australian version of The International Classification of Diseases, 9th Revision, Clinical Modification (ICD-9-CM). Sydney: National Coding Centre, 1996. i

\section{PREVENTION INTERVENTIONS FOR CHILD AND ADOLESCENT MENTAL HEALTH: NSW RESOURCE DOCUMENT}

\author{
Suzanne Pope, Beverley Raphael, and Kym Scanlon \\ Centre for Mental Health \\ NSW Department of Health
}

This article describes a new resource, Prevention initiatives for child and adolescent mental health: NSW Resource Document, produced by the Centre for Mental Health, that provides information on a range of programs designed to prevent the development of mental health problems and disorders in children and young people. Based on a literature review of the current research in prevention initiatives for child and adolescent mental health, it includes: initiatives aimed at the prevention of mental health problems, early intervention programs and services for young people with depression and related disorders, and first onset psychosis and suicide prevention.

\section{BACKGROUND}

Effective prevention programs have been identified which may help to reduce the risk of children developing a mental problem or disorder. Some prevention programs are even more effective than later treatments, particularly in the area of conduct disorders. Significant advancements can be made when both the early years of life and the early stages of disorders are targeted.
Mental health prevention and early intervention are relatively new fields in mental health. Progressing these initiatives involves supporting health and related staff and the community in the acquisition of the knowledge and skills needed to meet the challenges of new service directions and programs, including the provision of resources to assist implementation.

The NSW Department of Health is playing a leading role in national prevention initiatives in mental health, including the National Mental Health Promotion, Prevention and Early Intervention Action Plan, ${ }^{1}$ for the Second National Mental Health Plan. ${ }^{2}$ The NSW Resource Document will help to guide the implementation of the National Mental Health Reform Incentive Funding that has been provided to NSW over the next five years to help progress implementation of the Second National Mental Health Strategy.

\section{THE NSW RESOURCE DOCUMENT}

The NSW Resource Document is divided into eight sections:

Section 1 provides an introduction to the research and policies that have influenced the growing interest in evidence-based prevention initiatives for child and adolescent mental health. 